\title{
Combination of $\mathrm{t}(4 ; 14)$, del(17p13), del(1p32) and 1q21 gain FISH probes identifies clonal heterogeneity and enhances the detection of adverse cytogenetic profiles in 233 newly diagnosed multiple myeloma
}

\author{
Thomas Smol ${ }^{1,2,8^{*}} \mathbb{D}$, Annika Dufour ${ }^{3}$, Sabine Tricot ${ }^{4}$, Mathieu Wemeau ${ }^{5}$, Laure Stalnikiewicz ${ }^{6}$, Franck Bernardi ${ }^{7}$, \\ Christine Terré ${ }^{3}$, Benoît Ducourneau', Hervé Bisiau' ${ }^{1}$ and Agnès Daudignon'
}

\begin{abstract}
Background: Our aim was to set the FISH combination of del(17p13), t(4;14), 1 q21 gain and del(1p32), four adverse cytogenetic factors rarely evaluated together, and compare our technical thresholds with those defined in the literature.

Methods: Two hundred thirty-three patients with MM at diagnosis were studied using FISH to target 4 unfavorable cytogenetic abnormalities: 17p13 deletion, $\mathrm{t}(4 ; 14)$ translocation, $1 \mathrm{p} 32$ deletion and 1q21 gain. Technical thresholds were determined for each probe using isolated CD138-expressing PC from patients without MM.

Results: The FISH analysis identified abnormalities in $79.0 \%$ of patients. Del(17p13) was detected in $15.0 \%$ of cases, t(4;14) in $11.5 \%$, $1 \mathrm{q} 21$ gain in $37.8 \%$ and del(1p32) in $8.7 \%$. Adding 1p32/1q21 FISH probes has enabled us to identify adverse cytogenetic profiles in $39.0 \%$ of patients without del(17p13) or t(4;14). Clonal heterogeneity was observed in $51.1 \%$ of patients as well as an increase in the number of adverse abnormalities when related clones were greater than or equal to 2 (85.1\% against $45.6 \%)$.

Conclusion: FISH allowed detecting accumulation of adverse abnormalities and clonal heterogeneity in MM with a combination of 4 probes. The impacts of these two parameters need to be evaluated, and could be included in future cytogenetic classifications.
\end{abstract}

Keywords: Multiple Myeloma, Interphase FISH, 1q21 gain, 1p32 deletion, Thresholds

\section{Background}

Multiple Myeloma (MM) is a heterogeneous disease characterized by the clonal proliferation of abnormal plasma cells (PC), invading mainly the bone marrow (BM). MM accounts for approximately $1 \%$ of all cancers, and $10 \%$ of all hematopoietic neoplasms with a median age of 70 years at diagnosis [1]. The diagnosis of MM is

\footnotetext{
* Correspondence: thomas.smol@chru-lille.fr

'Service d'Hématologie-Immunologie-Cytogénétique, $\mathrm{CH}$ Valenciennes, Valenciennes, France

Université de Lille Nord de France, Lille, France

Full list of author information is available at the end of the article
}

based on BM infiltration by $10 \%$ or more clonal PC or dystrophic PC together with evidence of end organ damage [1].

Cytogenetic analysis plays a major role in the risk stratification of MM [2-4]. However, with a metaphase cytogenetic approach, only $35 \%$ of patients present abnormal karyotypes, often associated with an advanced stage of the disease [5]. Thus, practice guidelines now recommend interphase fluorescence in-situ hybridization (FISH) as the initial cytogenetic analysis for MM [6]. FISH is performed on isolated CD138-expressing plasma cells [7]. PC enrichment provides a pure tumour population that enables 
abnormalities to be detected, irrespective of proliferation and infiltration index.

The efficiency of the cell enrichment method limits the number of targets investigated by FISH and a selection of relevant probes is required to provide information on the diagnosis and prognosis. Many combinations of cytogenetic markers have been evaluated. Routine panels mainly evaluate the deletion of 17p13 (TP53 deletion) and the $\mathrm{t}(4 ; 14)(\mathrm{p} 16 ; \mathrm{q} 32)$ FGFR3-IGH translocation but do not cover the heterogeneity of MM. The $\operatorname{del}(17 \mathrm{p} 13)$ remains the most powerful cytogenetic factor regardless of the therapeutic choice, while $t(4 ; 14)$ loses its negative impact with proteasome inhibitors regimens [8]. Other rearrangements involving $I G H$ genes have been reported, such as $\mathrm{t}(14 ; 16)(\mathrm{q} 32 ; \mathrm{q} 23)$ and $\mathrm{t}(14 ; 20)(\mathrm{q} 32 ; \mathrm{q} 12)$. The prognostic values of the latter vary depending on working groups or methods used [9-11]. In contrast, partial aneuploidies of chromosome 1 (1q21 gain and 1p32 deletion) are retained as more relevant additional markers [10, 12], even with the emerging therapeutic approaches [13, 14]. The addition of 1q21 gain probe is beginning to be integrated into the FISH panel, whereas the identification of $1 \mathrm{p} 32$ deletion is not widely used. Moreover, studies are focused on the presence or the absence of FISH markers without considering the clone size or the number of clones.

Our aim was to set the FISH combination of $\operatorname{del}(17 \mathrm{p} 13), \mathrm{t}(4 ; 14), 1 \mathrm{q} 21$ gain and $\operatorname{del}(1 \mathrm{p} 32)$, four adverse cytogenetic factors rarely evaluated together. In a prospective study of 233 newly diagnosed MM, we observed that the proportion of patients with unfavourable cytogenetic markers could be extended, and that a high level of clonal heterogeneity could be identified by a sufficient number of FISH probes.

\section{Methods}

\section{Patient samples}

Between January 2013 and August 2015, BM samples were collected from 233 patients during diagnosis at the Cytogenetic Laboratories in Valenciennes General Hospital, and Versailles General Hospital. Patients were involved on the basis of WHO 2008 diagnostic criteria for MM. The institutional ethics committee approved the study.

\section{Plasma cell enrichment}

PC were enriched from BM mononuclear cells, using a magnetic cell-sorting CD138 MicroBeads kit (Miltenyi Biotec; BergischGlabach, Germany) according to the manufacturer's protocol. BM samples were filtered with a $70 \mu \mathrm{m}$ filter (Miltenyi Biotec), and suspended in RPMI solution (Dutscher; Brumath, France). After centrifugation $(160 \mathrm{G})$, and removal of the supernatant, mononuclear cells were marked with CD138 microbeads at $4^{\circ}$ $\mathrm{C}$ for $15 \mathrm{~min}$. The cells were then separated on a column kit separator (Miltenyi Biotec). PC enrichment was controlled on a cytospin slide by a cytologist. The median efficiency of CD138 selection was $97.5 \%$.

\section{Interphase fluorescent in situ hybridization}

FISH was performed on samples enriched in accordance with the manufacturer's instructions. The FISH panel included a TP53/CEP17 probe (Amplitech, Compiègne, France), a 1p32/CDKN2C-FAF1 - 1q21/CKS1B probe (Amplitech), a $\mathrm{t}(4 ; 14)(\mathrm{p} 16 ; \mathrm{q} 32)$ probe (MetaSystems, Altlussheim, Germany), and an IGH break-apart probe (MetaSystems).

An automated reading with images captured was performed on GSL-10 Leica (Leica Biosystems; Wetzlar, Germany) and analysed with CytoVision software (Leica Biosystems). The number of PC with abnormal signal patterns was calculated as the average of data from two cytogeneticists who analysed 200 cells. The quality of hybridization was controlled for each FISH technique on metaphases from negative samples.

Technical thresholds were determined for each probe, using isolated CD138-expressing $\mathrm{PC}$ from patients without MM, on the basis of the same method as used with patients with MM. Between five and ten PC controls were used for each probe. Thresholds were assessed after counting 200 cells for each negative sample, and established by a "mean + 3 DS" calculation. The technical thresholds for adverse cytogenetic abnormalities were $6.5 \%$ for $\operatorname{del}(1 \mathrm{p} 32), 4.5 \%$ for $1 \mathrm{q} 21$ gain, $4 \%$ for $\mathrm{t}(4 ; 14)$ translocation, $4 \%$ for $I G H$ rearrangement, and $5 \%$ for $\operatorname{del}(17 \mathrm{p} 13)$.

Compared technical thresholds from the literature were defined as $10 \%$ for fusions and $20 \%$ for numerical abnormalities [15].

\section{Statistical analysis}

A comparison of numerical variables between two groups was performed using the nonparametric MannWhitney $U$ test. A comparison of qualitative variables was performed using the Fisher $F$-test. Statistical significance was defined as $p<0.05$.

\section{Results}

Among the 233 patients, there were 52.3\% males ( $n=122)$ and $47.7 \%$ females $(n=111)$, with a median age of 67 years (41 to 89 years-old). The repartition of ISS score was respectively $37.6 \%, 33.6 \%$ and $28.8 \%$ for stage I, stage II, and stage III.

Cytogenetic abnormalities were identified in $79.0 \%$ of cases $(184 / 233)$ by FISH analysis (Table 1 ), with one or more adverse abnormalities in 51.9\% (121/233). We observed a del(17p13) in $15.0 \%$, a $\mathrm{t}(4 ; 14)$ translocation in $11.5 \%$, a $1 \mathrm{q} 21$ gain in $37.8 \%$, and a $\operatorname{del}(1 \mathrm{p} 32)$ in $8.7 \%$ of patients (Fig. 1). By adding the $1 \mathrm{p} 32 / 1 \mathrm{q} 21 \mathrm{FISH}$ probe, we were able to identify one or more adverse 
Table 1 Distribution of FISH abnormalities

\begin{tabular}{|c|c|}
\hline $\begin{array}{l}\text { Cytogenetic abnormalities [number of patients with available } \\
\text { data] }\end{array}$ & $\mathrm{n}[\%]$ \\
\hline \multicolumn{2}{|l|}{$\begin{array}{l}1 p 32 / C D K N 2 C-F A F 1-1921 / C K S 1 B \\
\text { probe }[n=230]\end{array}$} \\
\hline $1 \mathrm{q} 21$ gain $=3$ copies & $\begin{array}{l}87 \\
{[37.8]}\end{array}$ \\
\hline 1 q21 gain $>3$ copies & $\begin{array}{l}42 \\
{[18.3]}\end{array}$ \\
\hline 1 p32 monoallelic deletion & $19[8.3$ \\
\hline 1 p32 biallelic deletion & $1[0.4]$ \\
\hline monosomy 1 & $4[1.7]$ \\
\hline trisomy 1 & $7[3.0]$ \\
\hline \multicolumn{2}{|l|}{ TP53/CEP17 probe $[n=233]$} \\
\hline 17p13 monoallelic deletion & $\begin{array}{l}35 \\
{[15.0]}\end{array}$ \\
\hline monosomy 17 & $11[4.7]$ \\
\hline trisomy 17 & $\begin{array}{l}32 \\
{[13.7]}\end{array}$ \\
\hline trisomy 17 with one TP53 loss & $6[2.6]$ \\
\hline \multicolumn{2}{|l|}{$\begin{array}{l}\text { IGH break-apart and } \mathrm{t}(4 ; 14)(\mathrm{p} 16 ; \mathrm{q} 32) \\
\text { - IGH/FGFR3 probes }[n=217]\end{array}$} \\
\hline total $/ G H$ rearrangements & $\begin{array}{l}75 \\
{[34.6]}\end{array}$ \\
\hline $\mathrm{t}(4 ; 14)(p 16 ; q 32)$ & $\begin{array}{l}25 \\
{[11.5]}\end{array}$ \\
\hline monosomy 4 or 4 p16 deletion & $4[1.9]$ \\
\hline monosomy 14 or $14 q 32$ deletion & $12[5.2]$ \\
\hline IGHV loss & $\begin{array}{l}17 \\
{[12.8]}\end{array}$ \\
\hline $4 p 16$ gain & $\begin{array}{l}23 \\
{[10.9]}\end{array}$ \\
\hline 14q32 gain & $8[3.4]$ \\
\hline
\end{tabular}

Abbreviations: CDKN2C Cyclin-Dependent Kinase Inhibitor 2C, CEP17 Chromosome 17 centromere, CKS1B Cyclin-Dependent Kinases Regulatory Subunit 1, FAF1 FAS-associated Factor 1, FGFR3 Fibroblast Growth Factor Receptor 3, FISH fluorescence in-situ hybridization, IGH Immunoglobulin Heavy Locus, IGHv Immunoglobulin Heavy Locus variable region, TP53 Tumour Protein P53 abnormalities in $39.0 \%(64 / 164)$ of patients with an absence of TP53 deletion or $\mathrm{t}(4 ; 14)$. For these patients, a 1q21 gain and a monoallelic $\operatorname{del}(1 \mathrm{p} 32)$ were found respectively in $90.6 \%$ and $18.7 \%$ of cases. Both markers were associated in 6 cases.

When adverse cytogenetic abnormalities were present, they were isolated in $70.2 \%(85 / 121)$ and associated in $29.8 \%(36 / 121)$ of cases. In our cohort, statistically significant associations were observed between the presence of $1 \mathrm{q} 21$ gain and $t(4 ; 14)$, and between the presence of $\operatorname{del}(1 \mathrm{p} 32)$ and del(17p13) (Fig. 1). The 1q21 gain was present in $68.0 \%(17 / 25)$ of patients with $t(4 ; 14)$ translocation versus $35.1 \%(67 / 191)$ of patients without $\mathrm{t}(4 ; 14)(p=0.001)$. The $\operatorname{del}(1 \mathrm{p} 32)$ was found in $36.8 \%$ $(7 / 19)$ of patients with $\operatorname{del}(17 \mathrm{p} 13)$ versus $12.8 \%(27 / 211)$ of patients without del(17p13) $(p=0.01)$. A subgroup of 1q21 gain was identified with a number of 1q21 signals greater than 3 . Between 4 and 9 signals were observed in $18.3 \%$ of cases that could be considered as 1q21 amplifications. In our cohort, this subgroup was always found as a 1q21 gain side-line, emerging in $48.3 \%$ of cases. Derivative FISH abnormalities targeting 1p32, 1q21, 4p16, 14q32, 17p13 and D17Z1 loci were frequently identified and are listed in Table 1 for information.

The median sizes of identified adverse clones were $50 \%$ for $\operatorname{del}(17 \mathrm{p} 13), 80 \%$ for $\mathrm{t}(4 ; 14), 52 \%$ for $1 \mathrm{q} 21$ gain and $77.5 \%$ for del(1p32). By applying frequently used technical thresholds to our population (10\% for fusions and $20 \%$ for numerical abnormalities), $40 \%$ of $\operatorname{del}(17 \mathrm{p} 13)$ detected would have been considered as negative, as well as $24 \%$ of $1 \mathrm{q} 21$ gain, $16.5 \%$ of $\operatorname{del}(1 \mathrm{p} 32)$ and only $4 \%$ of $\mathrm{t}(4 ; 14)$. These under-detected clones were mainly represented by numerical abnormalities. The difference of FISH sensibilities may be explained by the probes' design with or without internal control.

Clonal heterogeneity with at least 2 related clones was observed in $51.1 \%(94 / 184)$ of cases. The cases with 3 or more related clones represented $13.6 \%$ (25/184) of the population. Adverse abnormalities were significantly

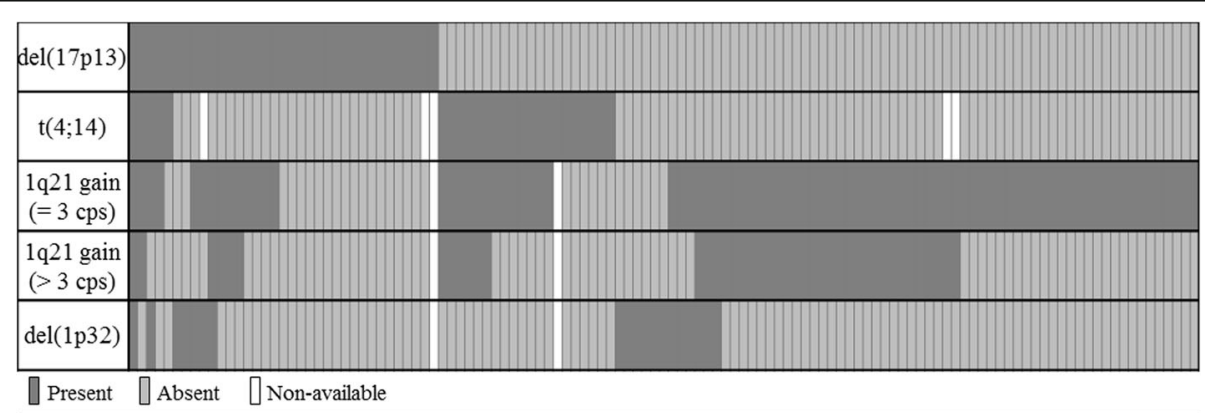

Fig. 1 Landscape of the association of adverse abnormalities at diagnosis $(n=121)$. Preferential associations were found between $t(4 ; 14)$ and $1 \mathrm{q} 21$ gain $(68.0 \%$ of cases in $\mathrm{t}(4 ; 14)$ subgroup), and between del(17p13) and del(1p32) (36.8\% of cases in del(17p13) subgroup). $1 \mathrm{q} 21$ amplification in more than 3 copies was found in $48.3 \%$ as 1 q21 gain side-line. (cps = copies) 
more frequent when the number of clones was greater than or equal to 2, with a frequency of $85.1 \%(80 / 94)$ against $45.6 \%(41 / 90)$ when 1 single clone was identified $(p<0.0001)$.

The distribution of adverse abnormality profiles varies with the number of identified clones (Fig. 2). When clonal heterogeneity was present ( $\geq 2$ clones), a greater number of MM with 1q21 gain were found: $81.6 \%$ (71/ 94) compared with $18.4 \%(16 / 88)$ when 1 single clone was identified $(p<0.0001)$. In the case of marked clonal heterogeneity ( $\geq 3$ clones), a higher involvement of $\operatorname{del}(1 \mathrm{p} 32)$ was found with a frequency of $28.0 \%(7 / 25)$ against 5.8\% (4/69) when only 2 related clones were present $(p=0.002)$. Del(17p13) and $\mathrm{t}(4 ; 14)$ were uniformly represented, regardless of the number of clones detected. A higher number of related clones tended to be observed when the ISS stage was III, with a median of 2 clones compared with a median of 1 clone when the ISS stages were I or II $(p=0.054)$.

\section{Discussion}

MM is characterized by the heterogeneity of cytogenetic changes, reflecting the heterogeneity of patients. Cytogenetic analyses have always played a key role in the prognostic evaluation in MM. However, low in-vitro PC proliferation index and low PC infiltration limited the conventional cytogenetic interpretation [5]. Culture failures or misinterpretations of normal karyotypes have been avoided with FISH on CD138-expressing PC [16]. In this study, we investigated $233 \mathrm{MM}$ during diagnosis with a combination of four probes targeting 17p13/TP53 deletion, $\mathrm{t}(4 ; 14)(\mathrm{p} 16 ; \mathrm{q} 32)$ translocation, $1 \mathrm{p} 32 / C D K N 2 C$ FAF1 deletion, and $1 \mathrm{q} 21 / C K S 1 B$ gain.

The prevalence of adverse abnormalities (51.9\%) in this cohort of $\mathrm{MM}$ at diagnosis is similar to that in previously published data $[2,3,12,16-18]$. The combination of unfavourable conventionally used cytogenetic markers, del(17p13) and $t(4 ; 14)$ [19], with two more recent markers, del(1p32) and 1q21 gain, has enabled us to identify $39.0 \%$ more patients carrying chromosome 1 abnormalities. Indeed, a predominance of 1q21 gain has been observed in $37.8 \%$ of cases, as well as a significant number of del(1p32) (8.3\%). The need to use a complete panel targeting these four markers in FISH can thus be confirmed.

Preferential associations between adverse abnormalities suggest a successive hits mechanism by analogy with the oncogene involvement in high-grade lymphomas. Several studies assess or stratify clinical risk by combining cytogenetic abnormalities with biomarkers. Two studies mention the accumulation of anomalies in MM with a score test based on the number of anomalies detected, combined with ISS stage [20, 21]. We observed that the accumulation of abnormalities is associated with clonal heterogeneity, considered as unfavourable in most hematologic malignancies. This parameter has not yet been integrated into prognostic models of various studies, even if a recent study indicates that the clone size affects patient outcomes [22]. As noted in previously published data, no association was observed between the number of adverse abnormalities and the ISS stage [11, 13]. Nevertheless, it seems that the clonal heterogeneity increases with the ISS stage III. This would support the idea of including clonal heterogeneity in a revised score.

The preceding remark raises the issue of how the thresholds of FISH defects are determined and used. The guidelines defined thresholds depending on the type of probe or thresholds based on prognostic switches. Such thresholds are either technical with $10 \%$ for fusions and $20 \%$ for numerical abnormalities [15], or prognostic from $60 \%$ up to $70 \%$ for $\operatorname{del}(17 \mathrm{p} 13)$, or from $10 \%$ up to $30 \%$ for $1 \mathrm{q} 21$ gain and $\mathrm{t}(4 ; 14)$ [12, 23-26]. In this study, we used our in-house establishment "mean +3 standard deviation" from normal isolated PC from BM. Our technical thresholds have enabled us to compare MM data from FISH with the same matrix as that used for the negative control. Thus, we were able to report all anomalies above these thresholds and identify all determinable

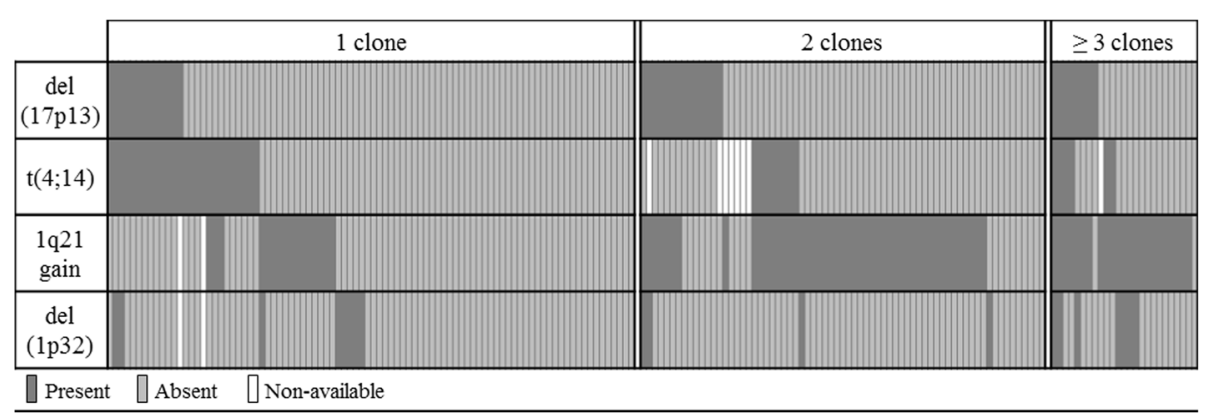

Fig. 2 Distribution of abnormalities according to the number of clones at diagnosis $(n=184)$. 1 q21 gain was more frequent in the subgroup of 2 clones or more (81.6\%) compared to the subgroup of 1 clone $(18.4 \%)(p<0.0001)$, del(1p32) was more frequent in the subgroup of 3 clones or more (28.0\%) compared to the subgroup of 1 or 2 clones $(5.8 \%)(p=0.002)$. No preferential distribution was observed with del(17p13) and $\mathrm{t}(4 ; 14)$ translocation 
clones with our four probes. Clonal heterogeneity would either have been less marked with previously published technical thresholds, or smoothed if prognostic thresholds were applied.

Detection of chromosome 1 abnormalities becomes relevant with the emergence of new therapies and therapeutic strategies. Indeed, it could identify $39.0 \%$ more patients with valuable prognostic information. Unlike $t(4 ; 14)$ whom negative impact can be erased by proteasome inhibitors [8], the 1q21 gain maintains a lower overall survival rate irrespective of treatment modality $[13,14]$. We observed that 1q21 gain was associated with a higher clonal heterogeneity at diagnosis. Moreover, the acquisition of this anomaly is often secondary, especially with $t(4 ; 14)$ translocation. Studies show the lack of 1q21 gain in monoclonal gammapathy of undetermined significance compared to $\mathrm{MM}$, suggesting a significant role of the 1q21 gain in MM progression [27, 28]. These data support the notion of $1 \mathrm{q} 21$ gain integration in the current cytogenetic classification in order to define therapeutic cytogenetic profiles, pending the establishment of consensual prognostic profiles.

The deletion of $1 \mathrm{p} 32$ locus is considered as a strong independent negative prognostic factor [12], and is associated with other adverse abnormalities in $2 / 3$ of cases in our series. $\operatorname{Del}(1 \mathrm{p} 32)$ potentiates the unfavourable characters of $\operatorname{del}(17 \mathrm{p} 13)$ or $\mathrm{t}(4 ; 14)$ translocation [12]. This deletion was identified in the presence of strong clonal heterogeneity ( $\geq 3$ related clones) reflecting a major chromosomal instability. It should therefore be a cytogenetic target in any FISH panel at MM diagnosis.

Cases with 2 or more trisomies are poorly represented in our series $(7.8 \%)$ (Table 1), while hyperdiploid MM represent approximately $50-55 \%$ of cytogenetic anomalies in MM $[29,30]$. This discordance arises because chromosomes 1, 4, 14 and 17 are poor indicators of hyperdiploidy in MM. Chromosomal gains affect preferentially the odd chromosomes 3, 5, 7, 9, 11, 15, 19 and 21 [31]. As a result, no conclusions about MM ploidy can be reached with our probes, unless we add a chromosomal centromeric marker among the most common trisomies, such as chromosomes 5, 9 or 15 in the set of FISH probes sorting [3, 29]. Cases with monosomies were rare, but consistent with published data, considering the non-use, in our cohort, of chromosome 13 probe known as the most frequent monosomy in MM [30].

\section{Conclusion}

The use of FISH on sorted PC has revolutionized the genetic analysis of MM. The absence of chromosomal banding analysis complicates the process of obtaining complete data concerning the combination of cytogenetic markers. However, in this study, we were able to identify adverse abnormalities or derivative anomalies, as well as related clones or clonal evolution by FISH analysis. We also confirm the presence of clonal heterogeneity and accumulation of adverse abnormalities in the first diagnostic analysis. The prognostic impact of these parameters should be evaluated, and could be included in future cytogenetic classifications.

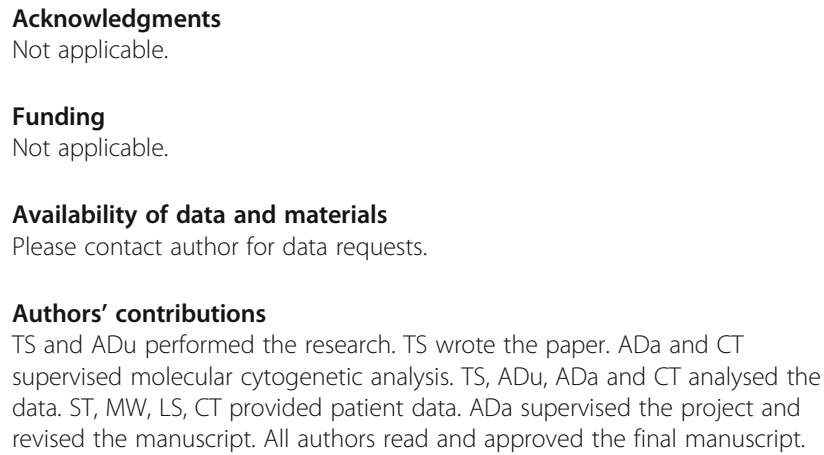

\section{Authors' contributions}

TS and ADu performed the research. TS wrote the paper. ADa and CT supervised molecular cytogenetic analysis. TS, ADu, ADa and CT analysed the data. ST, MW, LS, CT provided patient data. ADa supervised the project and revised the manuscript. All authors read and approved the final manuscript.

Ethics approval and consent to participate

The institutional ethics committee of Valenciennes general hospital and Versailles general hospital approved the study.

Consent for publication

Not applicable.

\section{Competing interests}

The authors declare that they have no competing interests.

\section{Publisher's Note}

Springer Nature remains neutral with regard to jurisdictional claims in published maps and institutional affiliations.

\section{Author details}

${ }^{1}$ Service d'Hématologie-Immunologie-Cytogénétique, CH Valenciennes, Valenciennes, France. ${ }^{2}$ Université de Lille Nord de France, Lille, France. ${ }^{3}$ Laboratoire de Cytogénétique Hématologique, CH Versailles, Le Chesnay, France. ${ }^{4}$ Service d'Hématologie Clinique, CH Valenciennes, Valenciennes, France. ${ }^{5}$ Service d'Hématologie Clinique, CH Arras, Arras, France. ${ }^{6}$ Service d'Hématologie Clinique, CH Lens, Lens, France. 'Laboratoire de Biologie, $\mathrm{CH}$ Douai, Douai, France. ${ }^{8}$ Institut de génétique médicale, Hôpital Jeanne de Flandre, CHRU Lille, Lille, France.

Received: 13 March 2017 Accepted: 25 June 2017

Published online: 01 July 2017

\section{References}

1. Vincent Rajkumar S. Multiple myeloma: 2014 Update on diagnosis, riskstratification, and management. Am J Hematol. 2014;89:999-1009.

2. Moreau P, Cavo M, Sonneveld P, Rosinol L, Attal M, Pezzi A, et al. Combination of international scoring system 3 , high lactate dehydrogenase, and $t(4 ; 14)$ and/or del(17p) identifies patients with multiple myeloma (MM) treated with front-line autologous stem-cell transplantation at high risk of early MM progression-related death. J Clin Oncol Off J Am Soc Clin Oncol. 2014;32:2173-80.

3. Hebraud B, Magrangeas F, Cleynen A, Lauwers-Cances V, Chretien M-L, Hulin $C$, et al. Role of additional chromosomal changes in the prognostic value of $t(4 ; 14)$ and del(17p) in multiple myeloma: the IFM experience. Blood. 2015;125:2095-100.

4. Leleu X, Karlin L, Macro M, Hulin C, Garderet L, Roussel M, et al. Pomalidomide plus low-dose dexamethasone in multiple myeloma with deletion 17p and/or translocation (4;14): IFM 2010-02 trial results. Blood. 2015;125:1411-7.

5. Liebisch P, Döhner H. Cytogenetics and molecular cytogenetics in multiple myeloma. Eur J Cancer Oxf Engl 1990. 2006:42:1520-9. 
6. Hastings R, Howell R, Betts D, Porter S, Haferlach C, Dastugue N, et al. A common European framework for quality assessment for banded chromosome studies and molecular cytogenetic investigations of acquired abnormalities. Eur Cytogenet Assoc Newsl. 2013;31:7-21.

7. Kobayashi S, Hyo R, Amitani Y, Tanaka M, Hashimoto C, Sakai R, et al. Fourcolor flow cytometric analysis of myeloma plasma cells. Am J Clin Pathol. 2006;126:908-15.

8. Avet-Loiseau H, Leleu X, Roussel M, Moreau P, Guerin-Charbonnel C, Caillot $D$, et al. Bortezomib plus dexamethasone induction improves outcome of patients with $t(4 ; 14)$ myeloma but not outcome of patients with del(17p). J Clin Oncol Off J Am Soc Clin Oncol. 2010;28:4630-4.

9. Avet-Loiseau H, Malard F, Campion L, Magrangeas F, Sebban C, Lioure B, et al. Translocation t(14;16) and multiple myeloma: is it really an independent prognostic factor? Blood. 2011;117:2009-11.

10. Kuiper $R$, van Duin $M$, van Vliet $M H$, Broijl $A$, van der Holt B, El Jarari $L$, et al. Prediction of high- and low-risk multiple myeloma based on gene expression and the International Staging System. Blood. 2015; 126:1996-2004.

11. Palumbo A, Avet-Loiseau H, Oliva S, Lokhorst HM, Goldschmidt H, Rosinol L, et al. Revised International Staging System for Multiple Myeloma: A Report From International Myeloma Working Group. J Clin Oncol Off J Am Soc Clin Oncol. 2015;33:2863-9.

12. Hebraud B, Leleu X, Lauwers-Cances V, Roussel M, Caillot D, Marit G, et al. Deletion of the 1 p32 region is a major independent prognostic factor in young patients with myeloma: the IFM experience on 1195 patients. Leukemia. 2014;28:675-9.

13. Nahi H, Våtsveen TK, Lund J, Heeg BMS, Preiss B, Alici E, et al. Proteasome inhibitors and IMiDs can overcome some high-risk cytogenetics in multiple myeloma but not gain 1q21. Eur J Haematol. 2015;96:46-54

14. An G, Xu Y, Shi L, Shizhen Z, Deng S, Xie Z, et al. Chromosome 1q21 gains confer inferior outcomes in multiple myeloma treated with bortezomib but copy number variation and percentage of plasma cells involved have no additional prognostic value. Haematologica. 2014;99:353-9.

15. Ross FM, Avet-Loiseau H, Ameye G, Gutiérrez NC, Liebisch P, O'Connor S, et al. Report from the European Myeloma Network on interphase FISH in multiple myeloma and related disorders. Haematologica. 2012;97:1272-7.

16. Bacher U, Haferlach T, Kern W, Alpermann T, Schnittger S, Haferlach C. Correlation of cytomorphology, immunophenotyping, and interphase fluorescence in situ hybridization in 381 patients with monoclonal gammopathy of undetermined significance and 301 patients with plasma cell myeloma. Cancer Genet Cytogenet. 2010;203:169-75.

17. Hillengass J, Zechmann CM, Nadler A, Hose D, Cremer FW, Jauch A, et al. Gain of 1q21 and distinct adverse cytogenetic abnormalities correlate with increased microcirculation in multiple myeloma. Int J Cancer. 2008;122:2871-5.

18. Nemec P, Zemanova Z, Kuglik P, Michalova K, Tajtlova J, Kaisarova P, et al. Complex karyotype and translocation $\mathrm{t}(4 ; 14)$ define patients with high-risk newly diagnosed multiple myeloma: results of CMG2002 trial. Leuk Lymphoma. 2012;53:920-7.

19. Dimopoulos M, Kyle R, Fermand J-P, Rajkumar SV, San Miguel J, ChananKhan A, et al. Consensus recommendations for standard investigative workup: report of the International Myeloma Workshop Consensus Panel 3. Blood. 2011;117:4701-5.

20. Avet-Loiseau H, Durie BGM, Cavo M, Attal M, Gutierrez N, Haessler J, et al. Combining fluorescent in situ hybridization data with ISS staging improves risk assessment in myeloma: an International Myeloma Working Group collaborative project. Leukemia. 2013;27:711-7.

21. Boyd KD, Ross FM, Chiecchio L, Dagrada GP, Konn ZJ, Tapper WJ, et al. A novel prognostic model in myeloma based on co-segregating adverse FISH lesions and the ISS: analysis of patients treated in the MRC Myeloma IX trial. Leukemia. 2012;26:349-55.

22. An G, Li Z, Tai Y-T, Acharya C, Li Q, Qin X, et al. The Impact of Clone Size on the Prognostic Value of Chromosome Aberrations by Fluorescence In Situ Hybridization in Multiple Myeloma. Clin Cancer Res. 2015;21:2148-56.

23. Avet-Loiseau H, Attal M, Moreau P, Charbonnel C, Garban F, Hulin C, et al. Genetic abnormalities and survival in multiple myeloma: the experience of the Intergroupe Francophone du Myélome. Blood. 2007;109:3489-95.

24. Neben $K$, Jauch A, Bertsch U, Heiss C, Hielscher T, Seckinger A, et al. Combining information regarding chromosomal aberrations $t(4 ; 14)$ and del(17p13) with the International Staging System classification allows stratification of myeloma patients undergoing autologous stem cell transplantation. Haematologica. 2010;95:1150-7.
25. Neben K, Lokhorst HM, Jauch A, Bertsch U, Hielscher T, van der Holt B, et al. Administration of bortezomib before and after autologous stem cell transplantation improves outcome in multiple myeloma patients with deletion 17p. Blood. 2012;119:940-8.

26. Chang $H$, Jiang $N$, Jiang $H$, Saha MN, Qi C, Xu W, et al. CKS1B nuclear expression is inversely correlated with p27Kip1 expression and is predictive of an adverse survival in patients with multiple myeloma. Haematologica. 2010;95:1542-7.

27. Hanamura I, Stewart JP, Huang Y, Zhan F, Santra M, Sawyer JR, et al. Frequent gain of chromosome band 1q21 in plasma-cell dyscrasias detected by fluorescence in situ hybridization: incidence increases from MGUS to relapsed myeloma and is related to prognosis and disease progression following tandem stem-cell transplantation. Blood. 2006;108:1724-32.

28. Sawyer JR, Tian E, Heuck CJ, Johann DJ, Epstein J, Swanson CM, et al. Evidence of an epigenetic origin for high-risk 1q21 copy number aberrations in multiple myeloma. Blood. 2015;125:3756-9.

29. Wuilleme S, Robillard N, Lodé L, Magrangeas F, Beris H, Harousseau J-L, et al. Ploidy, as detected by fluorescence in situ hybridization, defines different subgroups in multiple myeloma. Leukemia. 2005;19:275-8.

30. Van Wier S, Braggio E, Baker A, Ahmann G, Levy J, Carpten JD, et al. Hypodiploid multiple myeloma is characterized by more aggressive molecular markers than non-hyperdiploid multiple myeloma. Haematologica. 2013;98:1586-92.

31. Smadja NV. Bastard C, Brigaudeau C, Leroux D, Fruchart C, Groupe Français de Cytogénétique Hématologique. Hypodiploidy is a major prognostic factor in multiple myeloma. Blood. 2001;98:2229-38.

\section{Submit your next manuscript to BioMed Central and we will help you at every step:}

- We accept pre-submission inquiries

- Our selector tool helps you to find the most relevant journal

- We provide round the clock customer support

- Convenient online submission

- Thorough peer review

- Inclusion in PubMed and all major indexing services

- Maximum visibility for your research

Submit your manuscript at www.biomedcentral.com/submit
) Biomed Central 